\title{
THE SYNTHESIS MECHANISM OF CHLOROGENIC ACID IN LEAVES OF EUCOMMIA ULMOIDES OLIVER
}

\author{
MA, Y. ${ }^{1}-$ LIU, Y. ${ }^{1}-$ YANG, P. ${ }^{1}-$ ZHANG, T. ${ }^{1}-$ WU, Y.$^{2 *}$ \\ ${ }^{1}$ School of Life Sciences, Henan University, Kaifeng 475004, China \\ ${ }^{2}$ School of Pharmacy, Henan University, Kaifeng 475004, China \\ *Corresponding author \\ e-mail: wuyijun@henu.edu.cn
}

(Received $3^{\text {rd }}$ Sep 2019; accepted $12^{\text {th }}$ Feb 2020)

\begin{abstract}
This study revealed intraday variation of chlorogenic acid (CGA) and synthesis mechanism in leaves of Eucommia ulmoides Oliver by investigating relation of CGA content in leaves and the light intensity. CGA was quantified by High performance liquid chromatography (HPLC) with diode array detection (DAD). Illumination intensity was determined by portable photosynthesis system. It was shown that CGA content in leaves enhanced with the increase of light intensity and the highest point of CGA content in leaves got behind the highest point of illumination intensity. The fact that dark spots of blue formazan appeared in leaves which had been illuminated by sun light and were treated with nitroblue tetrazolium (NBT) showed that sunlight induces $\mathrm{O}_{2}-$ production in leaves. In addition, CGA possessed higher antioxidant activity and free radical scavenging activity in vitro experiment. DPPH radical scavenging activity was $2.43 \pm 0.092$ times of vitamin C equivalents (molar ratio), and ABTS radical cation scavenging activity was $3.61 \pm 0.14$ time of trolox equivalents (molar ratio), and reducing powers was $3.40 \pm 0.11$ times of vitamin $\mathrm{C}$ equivalents (molar ratio). In plant cells, free radicals produced by photo oxidation can damage the cells, and however, CGA can protect cells by eliminating free radicals. This may be the mechanism of the synthesis of chlorogenic acid in plants.
\end{abstract}

Keywords: chlorogenic acid, Eucommia ulmoides Oliver, oxygen free radical

\section{Introduction}

Eucommia ulmoides Oliver (E. ulmoides) is a traditional medicinal plant of China, and is also one of the main sources of chlorogenic acid (CGA). The pharmacological functions of CGA have been studied extensively. Potentially beneficial properties to human such as antimicrobial, antiinflammatory, antioxidant, anticancer, antiviral, hepatoprotective activities, antihypertension and protection against UV radiation have been attributed to CGA in vitro (Bhattacharyya et al., 2014; Chen et al., 2010; Shao et al., 2015). In plants, CGA performs anti-oxidant functions, and increased CGA accumulation is also associated with increased UV-protection (Cle et al., 2008; Doring and Petersen, 2014; Mudge et al., 2016). In this paper, the effect of illumination intensity on produce of CGA in leaves of E. ulmoides was investigated.

The quantitative determination methods of CGA have been reported, including gradient HPLC method, new flow injection chemiluminescent method, nano-LC-ESI mass spectrometry and electrochemical sensor (Hu et al., 2009; Santos et al., 2011; Wang et al., 2007; Zhang et al., 2007). However, a rapid, reliable and convenient determination of CGA is required. High performance liquid chromatography (HPLC) with diode array detection (DAD), more common and less costly, currently represents the most prevalent and reliable analytical method for the characterization of compounds, which need to be simultaneously acquired of chromatograms at any wavelength accompanied by the absorption spectrum of each eluted band and verified 
of separation quality with peak purity analysis (Huang et al., 2015; Luo et al., 2011; Sriseadka et al., 2012; Zeraik and Yariwake, 2010). Consequently, a normally manageable HPLC-DAD process was proposed in this research.

\section{Materials and methods}

\section{Materials}

Fresh leaves of E. ulmoides were obtained in campus of Henan University in summer and ground into dry powder, followed by sieving through 200 mesh screen to control the particle size.

\section{Reagents}

1,1-diphenyl-2-picrylhydrazyl (DPPH) and 2,2'-azinobis-(3-ethylbenzthiazoline- 6sulphonate (ABTS) were purchased from Sigma Chemical Co. Trolox was purchased from MP Biomedicals. Nitroblue tetrazolium (NBT) and CGA were purchased from Bomei Biotechnology Co. Methanol was chromatographically reagent grade. All other chemicals used in this study were of analytical reagent grade.

\section{Determination of sunlight intensity}

Sunlight intensity and temperature of leaves were determined by portable photosynthetic apparatus (LI-6400XT, LICOR USA) in a clear day.

\section{Determination of CGA in leaves}

CGA in leaves was separated and quantified using a Waters HPLC (Milford, Massachusetts, USA) equipped with a Waters1525 pump, a Waters 2707 autosampler, and Waters diode array detector 2998. Breeze Software was used for the data processing. C18 column (Waters, $250 \times 4.6 \mathrm{~mm}$ i.d., $5 \mu \mathrm{m}$ particle size) was used to identify CGA. The chromatographic separation was operated with solvent A $(\mathrm{MeOH})$ and B (water). The separated flavonoid peaks were identified by comparing the retention time of individual standards. The optimum procedure elution used in this study was as follows: a gradient of $70-30 \%$ A $(0-15 \mathrm{~min}), 30-70 \% \mathrm{~A}(15-20 \mathrm{~min})$. The samples were injected automatically $(20.0 \mu \mathrm{L})$. The column and guard column were controled at $30{ }^{\circ} \mathrm{C}$ and a $1.0 \mathrm{~mL} \min ^{-1}$ flow rate was applied, and the CGA were scanned from 210 to $400 \mathrm{~nm}$ in HPLC.

\section{$\mathrm{O}_{2}-$ localization in situ}

Fresh leaves from control (dark-treated leaves) and light-treated leaves were excised and immersed in a $0.1 \%$ solution of nitroblue tetrazolium (NBT) in $50 \mathrm{mM}$ Kphosphate buffer (pH 6.4), containing $10 \mathrm{mM} \mathrm{Na-azide,} \mathrm{and} \mathrm{were} \mathrm{vacuum-infiltrated} \mathrm{for} \mathrm{5-10} \mathrm{min} \mathrm{and}$ illuminated until appearance of dark spots, characteristic of blue formazan precipitates. Leaves were bleached by immersing in boiling ethanol (Romero-Puertas et al., 2004).

\section{Analytical method of antioxidant capacity}

The antioxidant capacity of CGA was determined by the Thermo Multiskan GO (Thermo Fisher Scientific Oy, Finland) (Shown as Table 1). The free radical scavenging 
activity was evaluated using the 2.2-diphenyl-1-picrylhydrazil (DPPH) assay and the absorbance values were measured at $518 \mathrm{~nm}$ and converted into the percent antioxidant activity (AA) using the following formula: AA\% $=[$ (absorbance of the control absorbance of the sample) / absorbance of the control] $\times 100$, The Vitamin $\mathrm{C}$ equivalents (VCEs) values of CGA were calculated from a linear regression of the AA of Vitamin $\mathrm{C}$ (VC, ascorbic acid); the scavenging activity against 2,2'-azino-bis(3ethylbenzo thiazoline)-6-sulfonic acid (ABTS) radical cation was measured by the method that ABTS radical cation was produced directly by reacting ABTS solution with potassium persulfate and allowing the mixture to stand for 12-16 $\mathrm{h}$ in dark at the room temperature and prior to beginning the assay, and ABTS solution was diluted with methanol to an absorbance of at $734 \mathrm{~nm}$, and then sample solution was added to ABTS solution and read at $734 \mathrm{~nm}$ after mixture and $30 \mathrm{~min}$ incubation at room temperature, and then The trolox equivalents (TEs) values of CGA were calculated from a linear regression of the AA of trolox equivalents; the determination of reducing powers of CGA was changed according to the method described by Ozsoy: $0.1 \mathrm{ml}$ VC (20-100 $\mu \mathrm{g} / \mathrm{ml})$ and $0.1 \mathrm{ml} \mathrm{CGA}(20 \mu \mathrm{g} / \mathrm{ml})$ were mixed with an equal volume of $0.2 \mathrm{M}$ phosphate buffer ( $\mathrm{pH} 6.6)$ and $1 \%$ potassium ferricyanide and then incubated at $50{ }^{\circ} \mathrm{C}$ for $20 \mathrm{~min}$, and then $0.25 \mathrm{ml}$ of $1 \%$ trichloroacetic acid was added to the mixture to stop the reaction, and then the mixture was centrifuged at $3000 \mathrm{~g}$ for $10 \mathrm{~min}$, and the supernatant $(0.25 \mathrm{ml})$ was mixed with $0.25 \mathrm{ml}$ distilled water and $0.1 \% \mathrm{FeCl}_{3}(0.5 \mathrm{ml})$ and then the absorbance was measured at $700 \mathrm{~nm}$, and the VCEs values of CGA were calculated from a linear regression of the AA of Vitamin C (Du et al., 2009; Dudonne et al., 2009; Ma et al., 2017; Ozsoy et al., 2008; Reis et al., 2015; Zengin et al., 2015).

Table 1. Antioxidant capacity of chlorogenic acid (CGA)

\begin{tabular}{c|c|c|c|c}
\hline & $\begin{array}{c}\text { VCEs } \\
\text { (mass ratio) }\end{array}$ & $\begin{array}{c}\text { VCEs } \\
\text { (molar ratio) }\end{array}$ & $\begin{array}{c}\text { TEs } \\
\text { (mass ratio) }\end{array}$ & $\begin{array}{c}\text { TEs } \\
\text { (molar ratio) }\end{array}$ \\
\hline DPPH radical scavenging activity & $1.21 \pm 0.046$ & $2.43 \pm 0.092$ & - & - \\
ABTS radical cation scavenging activity & - & - & $2.55 \pm 0.099$ & $3.61 \pm 0.14$ \\
Reducing powers & $1.69 \pm 0.054$ & $3.40 \pm 0.11$ & - & - \\
\hline
\end{tabular}

VCEs: vitamin C equivalents; TEs: trolox equivalents

\section{Statistical analysis}

Results were described as means \pm standard deviation (SD) of three measurements. Statistical analysis was carried out using Student's t-test and significant difference was statistically considered at the level of $\mathrm{p}<0.05$.

\section{Result and discussion}

The CGA peaks were identified by their UV/DAD spectra due to their characteristic UV spectral pattern (Band I, $\lambda$ max around $320 \mathrm{~nm}$ and Band II, $\lambda$ max around $220 \mathrm{~nm}$ ) in Figure 1. The absorption spectrum of each eluted band of leaves of E. ulmoides at any wavelength was consistent with that of CGA standard (Fig. $1 a$ and $b$ ). Practically, UV/DAD scanning of herbal medicines without grinding makes CGA determination much more convenient. 

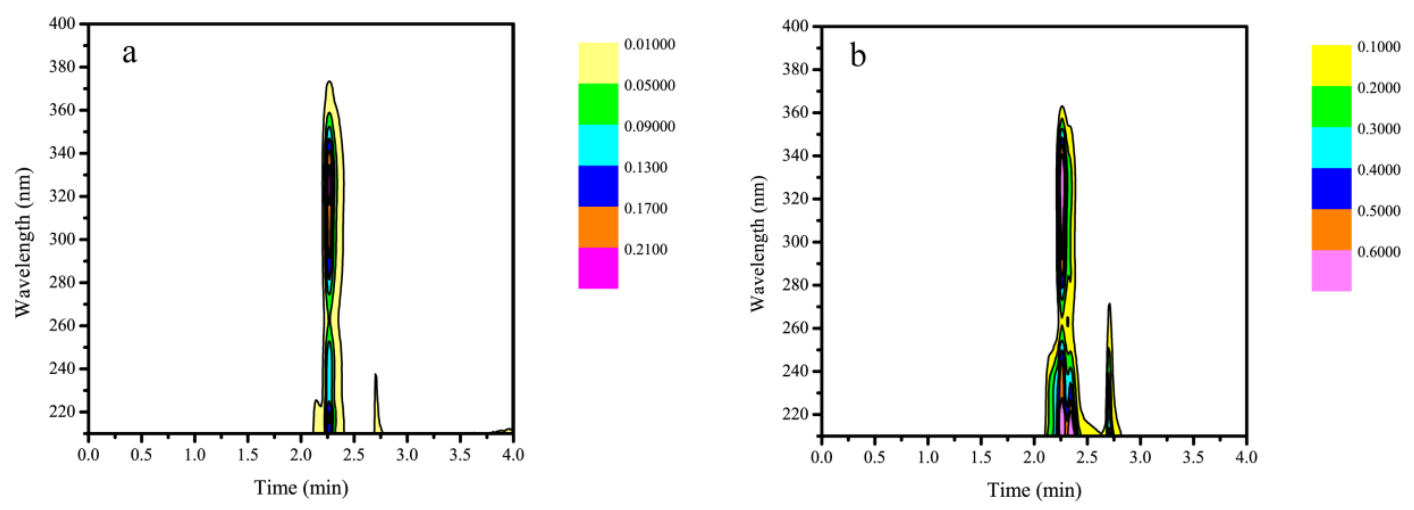

Figure 1. HPLC-UV/DAD of chlorogenic acid (CGA). a: CGA standard, b: leaves of E. ulmoides

Under clear day solar radiation condition, the change of CGA content in leaves of $E$. ulmoides with the illumination intensity was shown in Figure 2. The highest CGA content in leaves appeared at the decrease point of illumination intensity (14:00) but not the top of illumination intensity (12:00). And then CGA content in leaves dropped fast with illumination intensity after 14:00.

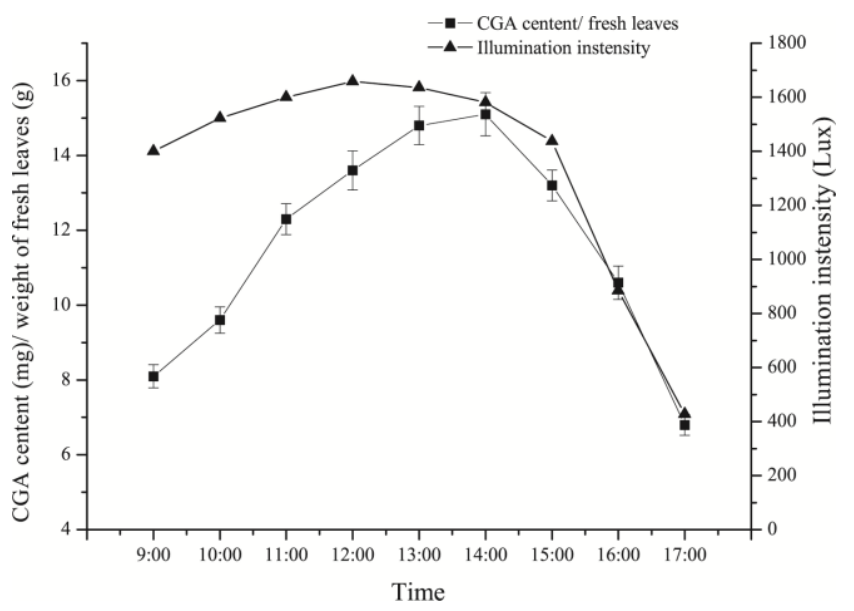

Figure 2. Intraday variation of chlorogenic acid (CGA) content in leaves of E. ulmoides

Fresh leaves of E. ulmoides had been illuminated by sun light and then were treated with nitroblue tetrazolium (NBT). In Figure 3, the dark spots of blue formazan appeared in leaves, while no dark spots appearing in the control (leaves in dark). This showed that sunlight induces $\mathrm{O}_{2}$ - production in leaves.

CGA performed higher antioxidant capacity: DPPH radical scavenging activity was $2.43 \pm 0.092$ times of VCEs $(\mathrm{mmol} / \mathrm{mmol})$, and ABTS radical cation scavenging activity was $3.61 \pm 0.14$ time of TEs $(\mathrm{mmol} / \mathrm{mmol})$, and reducing powers was $3.40 \pm 0.11$ times of VCEs $(\mathrm{mmol} / \mathrm{mmol})$.

In plant, reactive oxygen species (ROS) are involved in plant responses to various biotic and abiotic stresses (Ma et al., 2012; Wang et al., 2013; Qi et al., 2018). Plants requires light for photosynthesis, but absorption of too much light can lead to photooxidative damage to the photosynthetic apparatus, and then this phenomenon occurs due 
to imbalances which result from the overproduction of ROS (Chung et al., 2015; Ramabulana et al., 2016). However, accumulation of ROS can damage various cellular components such as proteins, nucleic acids, carbohydrates, and unsaturated lipids (Yu et al., 2018). Here, increased illumination intensity accelerated photooxidation reaction rate, which lead to enhancement of ROS level in cells. And then ROS heightened the content of CGA in the cell by increasing the expression of the genes related to the biosynthesis of CGA, which was completed over a period of time. Probably, this was why the top CGA content in leaves was late for a few hours than illumination intensity.
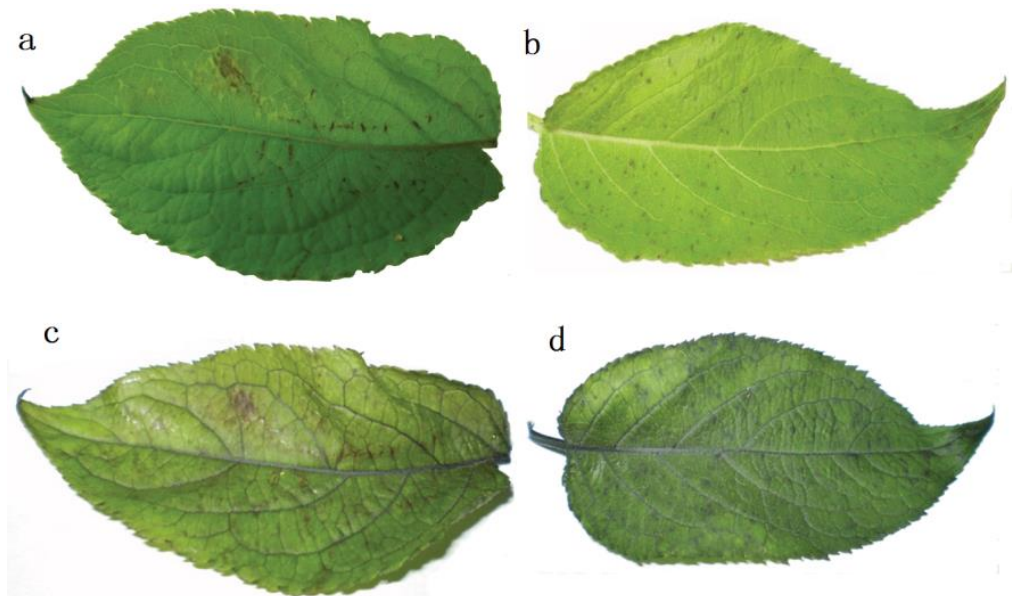

Figure 3. $\mathrm{O}_{2}-$ localization in situ of fresh leaves of E. ulmoides. Control leaf and sample leaf illuminated by sun light were treated with NBT and then bleached by immersing in boiling ethanol; a: control leaf; $b$ : sample leaf c: control leaf treated by boiling ethanol; d: sample leaf treated by boiling ethanol

In plant cells, free radicals produced by photo oxidation can damage the cells, and however, CGA can eliminate free radicals, which can protect cells. This may be the mechanism of the synthesis of chlorogenic acid in plants.

Acknowledgements. The authors are grateful to Zhiqi Cao, Yuanlong $\mathrm{Li}$ and Zhen Yang for the preparation work of this study. Scientific Research Fund of Henan Provincial Education Department (12B180004).

\section{REFERENCES}

[1] Bhattacharyya, S., Majhi, S., Saha, B. P., Mukherjee, P. K. (2014): Chlorogenic acidphospholipid complex improve protection against UVA induced oxidative stress. Journal of Photochemistry and Photobiology B-Biology 130: 293-298.

[2] Chen, X. M., Sang, X. X., Li, S. H., Zhang, S. J., Bai, L. H. (2010): Studies on a chlorogenic acid-producing endophytic fungi isolated from Eucommia ulmoides Oliver. Journal of Industrial Microbiology \& Biotechnology 37(5): 447-454.

[3] Chung, V. C. H., Ho, R. S. T., Wu, X., Fung, D. H. Y., Lai, X., Wu, J. C. W., Wong, S. Y. S. (2015): Are meta-analyses of Chinese herbal medicine trials trustworthy and clinically applicable? A cross-sectional study. - Journal of Ethnopharmacology 162(0): 47-54. 
[4] Cle, C., Hill, L. M., Niggeweg, R., Martin, C. R., Guisez, Y., Prinsen, E., Jansen, M. A. K. (2008): Modulation of chlorogenic acid biosynthesis in Solanum lycopersicum; consequences for phenolic accumulation and UV-tolerance. - Phytochemistry 69(11): 2149-2156.

[5] Doring, A. S., Petersen, M. (2014): Production of caffeic, chlorogenic and rosmarinic acids in plants and suspension cultures of Glechoma hederacea. - Phytochemistry Letters 10: CXI-cxvii.

[6] Du, G., Li, M., Ma, F., Liang, D. (2009): Antioxidant capacity and the relationship with polyphenol and Vitamin C in Actinidia fruits. - Food Chemistry 113(2): 557-562.

[7] Dudonne, S., Vitrac, X., Coutiere, P., Woillez, M., Merillon, J.-M. (2009): Comparative study of antioxidant properties and total phenolic content of 30 plant extracts of industrial interest using DPPH, ABTS, FRAP, SOD, and ORAC assays. - Journal of Agricultural and Food Chemistry 57(5): 1768-1774.

[8] Hu, F. L., Deng, C. H., Liu, Y., Zhang, X. M. (2009): Quantitative determination of chlorogenic acid in Honeysuckle using microwave-assisted extraction followed by nanoLC-ESI mass spectrometry. - Talanta 77(4): 1299-1303.

[9] Huang, R. T., Lu, Y. F., Inbaraj, B. S., Chen, B. H. (2015): Determination of phenolic acids and flavonoids in Rhinacanthus nasutus (L.) Kurz by high-performance-liquidchromatography with photodiode-array detection and tandem mass spectrometry. Journal of Functional Foods 12(0): 498-508.

[10] Luo, C., Zou, X., Li, Y., Sun, C., Jiang, Y., Wu, Z. (2011): Determination of flavonoids in propolis-rich functional foods by reversed phase high performance liquid chromatography with diode array detection. - Food Chemistry 127(1): 314-320.

[11] Ma, L. Y., Zhang, H., Sun, L. R., Jiao, Y. H., Zhang, G. Z., Miao, C., Hao, F. S. (2012): $\mathrm{NADPH}$ oxidase AtrbohD and AtrbohF function in ROS-dependent regulation of $\mathrm{Na}+/ \mathrm{K}+$ homeostasis in Arabidopsis under salt stress. - Journal of Experimental Botany 63(1): 305-317.

[12] Ma, Y., Wu, Y., Yang, J., Liang, H., Shang, F. (2017): Flavonoids content and antioxidant activity of ethanol extracts of Osmanthus fragrans flowers. - Bangladesh Journal of Botany 46(3): 907-915.

[13] Mudge, E., Applequist, W. L., Finley, J., Lister, P., Townesmith, A. K., Walker, K. M., Brown, P. N. (2016): Variation of select flavonols and chlorogenic acid content of elderberry collected throughout the Eastern United States. - Journal of Food Composition and Analysis 47: 52-59.

[14] Ozsoy, N., Can, A., Yanardag, R., Akev, N. (2008): Antioxidant activity of Smilax excelsa L. leaf extracts. - Food Chemistry 110(3): 571-583.

[15] Qi, J. S., Song, C. P., Wang, B. S., Zhou, J. M., Kangasjarvi, J., Zhu, J. K., Gong, Z. Z. (2018): Reactive oxygen species signaling and stomatal movement in plant responses to drought stress and pathogen attack. - Journal of Integrative Plant Biology 60(9): 805-826.

[16] Ramabulana, T., Mavunda, R. D., Steenkamp, P. A., Piater, L. A., Dubery, I. A., Madala, N. E. (2016): Perturbation of pharmacologically relevant polyphenolic compounds in Moringa oleifera against photo-oxidative damages imposed by gamma radiation. Journal of Photochemistry and Photobiology B-Biology 156: 79-86.

[17] Reis, L. C. B., Carneiro, L. M., Branco, C. R. C., Branco, A. (2015): Comparison of conventional microwave and focused microwave-assisted extraction to enhance the efficiency of the extraction of antioxidant flavonols from jocote pomace (Spondias purpurea L.). - Plant Foods for Human Nutrition 70(2): 160-169.

[18] Romero-Puertas, M. C., Rodriguez-Serrano, M., Corpas, F. J., Gomez, M., Del Rio, L. A., Sandalio, L. M. (2004): Cadmium-induced subcellular accumulation of O-2(.-) and $\mathrm{H} 2 \mathrm{O} 2$ in pea leaves. - Plant Cell and Environment 27(9): 1122-1134.

[19] Santos, W. D. R., Santhiago, M., Yoshida, I. V. P., Kubota, L. T. (2011): Novel electrochemical sensor for the selective recognition of chlorogenic acid. - Analytica Chimica Acta 695(1-2): 44-50. 
[20] Shao, P., Zhang, J. F., Chen, X. X., Sun, P. L. (2015): Microwave-assisted extraction and purification of chlorogenic acid from by-products of Eucommia ulmoides Oliver and its potential anti-tumor activity. - Journal of Food Science and Technology-Mysore 52(8): 4925-4934.

[21] Sriseadka, T., Wongpornchai, S., Rayanakorn, M. (2012): Quantification of flavonoids in black rice by liquid chromatography-negative electrospray ionization tandem mass spectrometry. - Journal of Agricultural and Food Chemistry 60(47): 11723-11732.

[22] Wang, P. C., Du, Y. Y., Zhao, X. L., Miao, Y. C., Song, C. P. (2013): The MPK6-ERF6ROS-responsive cis-acting element7/GCC box complex modulates oxidative gene transcription and the oxidative response in Arabidopsis. - Plant Physiology 161(3): 13921408.

[23] Wang, X. X., Wang, J. W., Yang, N. J. (2007): Chemiluminescent determination of chlorogenic acid in fruits. - Food Chemistry 102(1): 422-426.

[24] Yu, J. J., Zhang, Y. X., Liu, J. M., Wang, L., Liu, P. P., Yin, Z. P., Guo, S. Y., Ma, J., Lu, Z., Wang, T., She, Y. M., Miao, Y. C., Ma, L., Chen, S. X., Li, Y., Dai, S. J. (2018): Proteomic discovery of $\mathrm{H} 2 \mathrm{O} 2$ response in roots and functional characterization of PutGLP gene from alkaligrass. - Planta 248(5): 1079-1099.

[25] Zengin, G., Uysal, S., Ceylan, R., Aktumsek, A. (2015): Phenolic constituent, antioxidative and tyrosinase inhibitory activity of Ornithogalum narbonense L. from Turkey: a phytochemical study. - Industrial Crops and Products 70: 1-6.

[26] Zeraik, M. L., Yariwake, J. H. (2010): Quantification of isoorientin and total flavonoids in Passiflora edulis fruit pulp by HPLC-UV/DAD. - Microchemical Journal 96(1): 86-91.

[27] Zhang, Q. L., Li, J., Wang, C., Sun, W., Zhang, Z. T., Cheng, W. M. (2007): A gradient HPLC method for the quality control of chlorogenic acid, linarin and luteolin in Flos Chrysanthemi Indici suppository. - Journal of Pharmaceutical and Biomedical Analysis 43(2): 753-757. 